\title{
Autophagy in cultured murine neurons infected with equid herpesvirus 1
}

\author{
J. CYMERYS ${ }^{1}$, D. MISZCZAK ${ }^{1}$, A. SŁOŃSKA², A. GOLKE ${ }^{1}$ M. W. BAŃBURA ${ }^{1}$
}

\begin{abstract}
${ }^{1}$ Division of Microbiology, Department of Preclinical Sciences, Faculty of Veterinary Medicine, Warsaw University of Life Sciences SGGW, Ciszewskiego 8, 02-786 Warsaw, Poland; ${ }^{2}$ Division of Physiology, Department of Physiological Sciences, Faculty of Veterinary Medicine, Warsaw University of Life Sciences - SGGW, 02-776 Warsaw, Poland
\end{abstract}

Received October 25, 2013; accepted August 7, 2014

\begin{abstract}
Summary. - In this study we investigated the relationship of equid herpesvirus 1 (EHV-1) infection to autophagy in primary culture of murine neurons. Infection with both Jan-E and Rac-H strains of EHV-1 resulted in the formation of autophagosomes in the cytoplasm during early stages of infection, while in late stages of infection autophagosomes were mainly concentrated around the nucleus what suggests the induction of nuclear envelope-derived autophagy (NEDA). No significant effect of an authophagy inhibitor-chloroquine on final virus titers demonstrated that autophagy is not essential for EHV-1 replication.
\end{abstract}

Keywords: equid herpesvirus 1; autophagy; murine neurons; chloroquine

\section{Introduction}

Autophagy is a highly conserved self-degradation process of cellular components. Viral infections are one of many external factors that induce autophagy in host cells. During viral infections autophagy participates in the degradation of infectious virus particles within autophagolysosome in order to restrict viral replication and spread as well as providing ligands (viral components) for endosomal Toll-like receptors important for induction of innate immunity (Cavignac and Esclatine, 2010). There is an evidence that autophagy may be utilized by certain viruses to support their replication or facilitate viral particle egress. Over the past 10 years it has been demonstrated that herpesviruses developed various strategies to control the host autophagic machinery (Cavignac and Esclatine, 2010). In analyzing autophagy in the context of Herpesviridae infectivity consideration should be given to the fact, that the process may be accompanied

E-mail: jcymerys@op.pl; phone: +4822-5936060.

Abbreviations: EHV-1 = equid herpesvirus 1; HHV-1 = human herpesvirus 1; HHV-5 = human herpesvirus 5; NEDA = nuclear envelope derived autophagy; p.i. = post infection by productive infection as well as the latent one. Human herpesvirus 1 (HHV-1), a member of the Alphaherpesvirinae subfamily, is well known for its interference with the autophagic machinery.

Our study model focused on the involvement of autophagy during replication of another member of the Alphaherpesvirinae subfamily - equid herpesvirus 1 (EHV-1). Despite the fact that EHV-1 remains one of the most important respiratory viral pathogens affecting horses worldwide, there are still many open questions regarding the relationship of autophagy to EHV-1 replication. Moreover, to date there are no reports on such subject.

\section{Materials and Methods}

Virus strains. In this study two strains of EHV-1 from the virus collection of the Virology Laboratory of the Department of Preclinical Sciences were used. Jan-E is a field strain isolated from aborted fetus (mare Ezelda, Janow Podlaski stud, Poland; $12^{\text {th }}$ passage in ED cells) and identified by PCR using gB-specific primers (Borchers and Slater, 1993). Rac-H is a reference strain isolated from mare Heraldia, Racot stud, Poland, which has been passaged through a series of cell cultures, and described as 'pantropic', nonpathogenic (Nugent et al., 2006). 

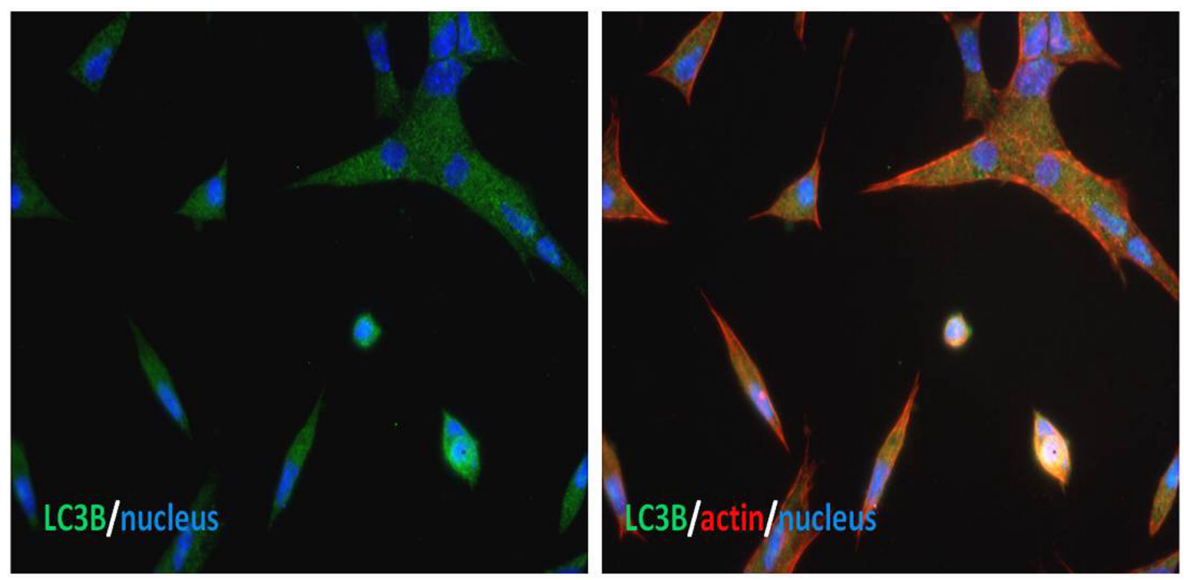

Fig. 1

Autophagosomes in murine neurons Fluorescent microscopy of non-infected murine neurons. Autophagosomes (LC3B, green), actin (red) and nuclei (blue). Magnification 400x.
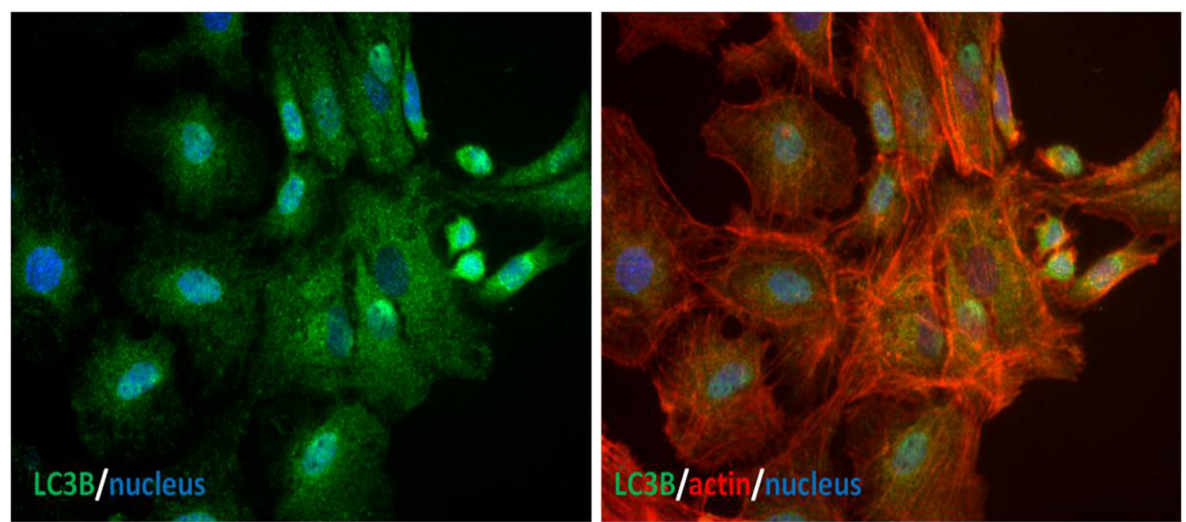

Fig. 2

Autophagosomes in murine neurons exposed to chloroquine for $16 \mathrm{hr}$ Fluorescent microscopy of non-infected murine neurons. Autophagosomes (LC3B, green), actin (red) and nuclei (blue). Magnification 400x.
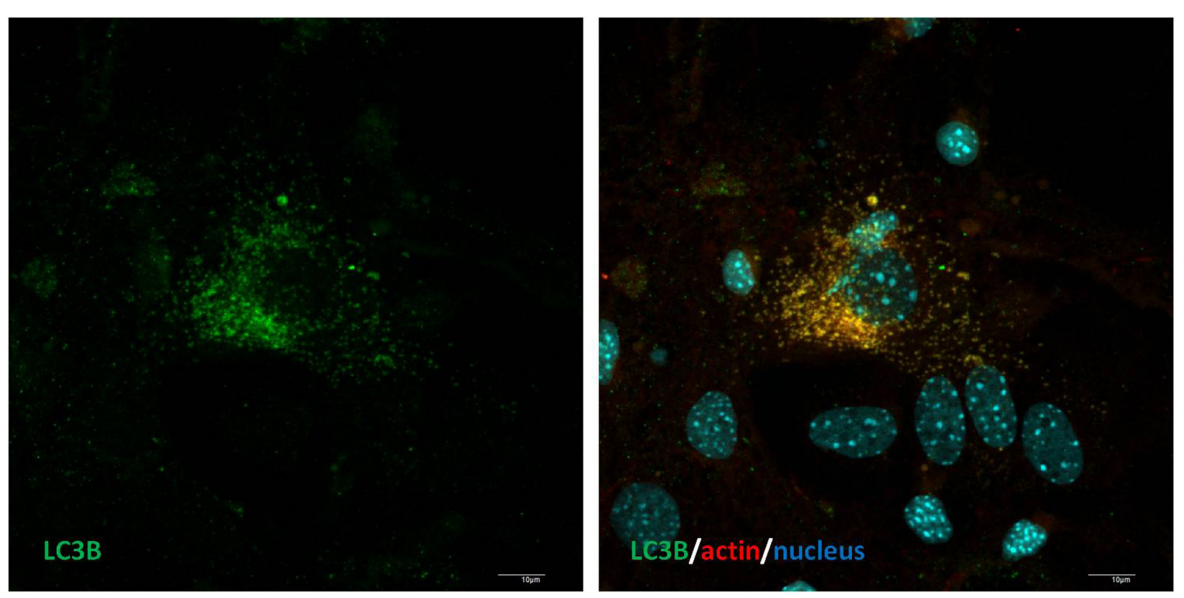

Fig. 3

Autophagosomes in murine neurons infected with EHV-1 Jan-E strain Fluorescent microscopy $2 \mathrm{hr}$ p.i. Autophagosomes (LC3B, green), actin (red) and nuclei (blue). Magnification 400x.
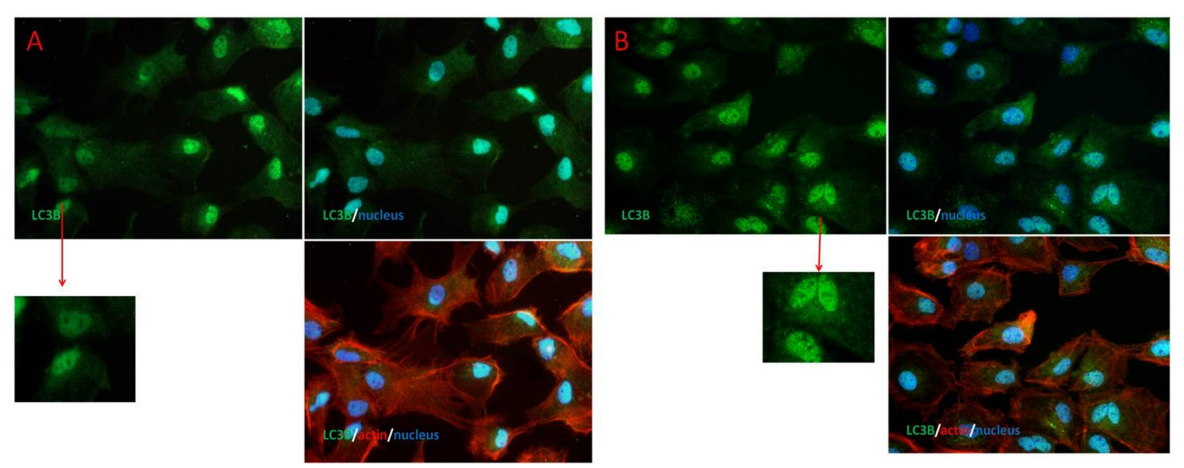

Fig. 4

Autophagosomes in murine neurons infected with Rac-H (a) and Jan-E (b) strains of EHV-1

Fluorescent microscopy $24 \mathrm{hr}$ p.i. Autophagosomes (LC3B, green), actin (red) and nuclei (blue). Magnification 400x. 
Cells. Balb/c (H-2 $)$ mice genetically susceptible to EHV-1 infection were used to establish primary culture of murine neurons, as described before (Cymerys et al., 2010). Cells were plated onto poly-L-lysine or poly-D-lysine with laminin-coated coverslips at a density of $5 \times 10^{4}$ neurons per well $\left(3.6 \mathrm{~cm}^{2}\right)$. Primary murine neurons were cultured in B-27 Neuron plating medium, consisting of neurobasal medium, B-27 supplement, $200 \mathrm{mmol} / \mathrm{l}$ of glutamine, $10 \mathrm{mmol} / \mathrm{l}$ of glutamate and penicillin/streptomycin antibiotics with $10 \%$ supplement of fetal bovine (5\%) and equine serum (5\%) (Gibco Life Technologies). Cultures were maintained at $37^{\circ} \mathrm{C}$ in $5 \% \mathrm{CO}_{2}$. Primary murine neuron cultures $\left(10^{5}\right.$ cells per well $/ 3.6$ $\mathrm{cm}^{2}$ ) were infected with each EHV-1 strain (MOI 1.0) for $60 \mathrm{~min}$ at $37^{\circ} \mathrm{C}$. After adsorption, the inoculum was aspirated and fresh culture medium was added. Then the cells were incubated for 2 and $24 \mathrm{hr}$ at $37^{\circ} \mathrm{C}$ with $5 \% \mathrm{CO}_{2}$.

Autophagy detection. Rabbit polyclonal antibody-LC3B (Gibco Life Technologies; $0.5 \mu \mathrm{g} / \mathrm{ml}$ ), was used to detect authophagy and visualised with secondary Alexa Fluor 488 goat anti-rabbit IgG MAb (Sigma-Aldrich; dilution 1:200). Actin filament structures were visualised by TRITC-phalloidin conjugate (Sigma-Aldrich; $500 \mathrm{ng} / \mathrm{ml}$ ). Cell nuclei were stained with bisbenzimidine/Hoechst 33258 (Sigma-Aldrich; $2 \mu \mathrm{g} / \mathrm{ml}$ ), in compliance with manufacturers recommendations. A positive control for autophagy was prepared by adding chloroquine diphosphate (Gibco Life Technologies; 5 $\mu \mathrm{mol} / \mathrm{ml}$ ), for artificially generating autophagosomes. Following chloroquine diphosphate treatment ( $16 \mathrm{hr}$ incubation in neuron culture), lysosomal $\mathrm{pH}$ increases and the normal autophagic flux is disrupted, resulting in autophagosome accumulation. Non-infected neurons served as negative control. Results were evaluated under fluorescence (Olympus BX60) and confocal microscope (Fluoview FV10i, Olympus).

Real-time PCR. The quantity of EHV-1 DNA in all samples (non-infected neurons, neurons infected with EHV-1 and neurons treated for $16 \mathrm{hr}$ with $5 \mu \mathrm{mol} / \mathrm{ml}$ chloroquine diphosphate after EHV-1 infection), was estimated using real-time PCR technique with fluorescent TaqMan probe, complementary for the sequence within the amplified product. Tests were done on the LightCycler 2.0 instrument (Roche Diagnostics), using an in-house quantitative method (Dzieciątkowski et al., 2009). Jan-E EHV-1 strain serial dilutions were used as reaction standards and DNA from non-infected ED cells served as negative control. Each sample was amplified with internal control (positive control of the amplification process), and tested in the independent duplicates.

Statistical analysis. Each analysis was carried out at least in triplicate, and each experiment was repeated at least twice. Differences in means with $\mathrm{P} \leq 0.05$ were considered significant (Student's $t$-test).

\section{Results and Discussion}

Immunofluorescence staining of primary murine neurons infected with Jan-E and Rac-H EHV-1 strains demonstrated that autophagosomes were formed at an early stage of infection ( $2 \mathrm{hr}$ post infection (p.i.)). We observed an increase of autophagosome accumulation mostly in the cytoplasm of infected neurons (Fig. 3). Twenty-four hr post EHV-1 infection smaller number of autophagosomes was found within the cytoplasm of primary murine neurons, whereas intense fluorescence was observed around the nucleus (Fig. 4). Incubation with chloroquine for $16 \mathrm{hr}$ (positive control), resulted in an accumulation of autophagosomes visible in the form of intensive green fluorescence (Fig. 2) and the signal was emitted mostly from the cytoplasmic region, which was not observed in uninfected cells (negative control) (Fig.1). Real-time PCR analysis showed that there was no influence of chloroquine on
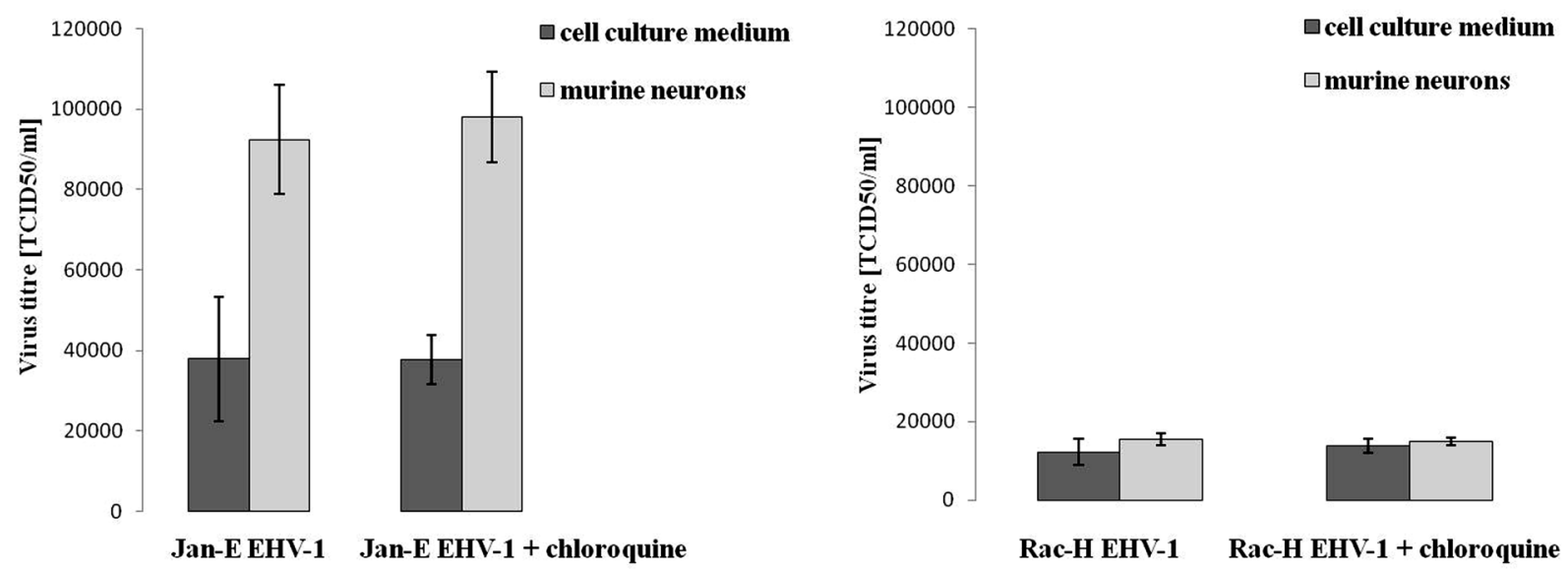

Fig. 5

Virus titers in murine neurons infected with Jan-E and Rac-H strains of EHV-1 exposed to chloroquine for $16 \mathrm{hr}$ Real time PCR 24 hr p.i. 
the replication of both tested EHV-1 strains. The number of copies of virus genome in murine neuronal cells and in culture medium were not significantly different both in control and after treatment with chloroquine (Fig. 5).

Recent experimental data show that autophagy induction may occur in many ways, during different stages of infection. Some viruses can induce autophagy by the mere presence of viral DNA in a cell, independently of viral protein synthesis (McFarlane et al., 2011; Rasmussen et al., 2011). It has been demonstrated that early induction of autophagy in cells infected with HHV-1 and HHV-5 was elicited by the presence of extraneous DNA (McFarlane et al., 2011). This scenario is highly probable during EHV-1 infection as well. Additionally, at a late stage of $\mathrm{HHV}-1$ infection nuclear envelope-derived autophagy (NEDA) has been discovered (Radtke et al., 2013). Thus we speculate, that NEDA may be also a response to EHV-1 infection. Despite autophagy is a mechanism that usually affects virus replication, we did not observed any effect of chloroquine on EHV-1 replication in primary culture of murine neurons. Our study has provided some answers to a number of key questions relating to induction of neuronal autophagy by EHV-1. Nevertheless, the influence of autophagy on EHV-1 replication is controversial and requires further research.

Acknowledgements. This work was supported by the grant No. NN308 028739 from Polish Ministry of Science and Higher Education.

\section{References}

Borchers K, Slater J (1993): A nested PCR for the detection and differentiation of EHV-1 and EHV-4. J. Virol. Methods
45, 331-336. http://dx.doi.org/10.1016/0166-0934(93)90117-A

Cavignac Y, Esclatine A (2010): Herpesviruses and autophagy: catch me if you can! Viruses 2, 314-333. http://dx.doi. org/10.3390/v2010314

Cymerys J, Dzieciątkowski T, Słońska A, Bierła J, Tucholska A, Chmielewska A, Golke A Bańbura MW (2010): Equine herpesvirus type 1 (EHV-1) replication in primary murine neurons culture. Pol. J. Vet. Sci. 13, 701-708. http:// dx.doi.org/10.2478/v10181-010-0022-3

Dzieciątkowski T, Przybylski M, Cymerys J, Turowska A, Chmielewska A, Tucholska A, Bańbura MW (2009): Equine herpesvirus type 1 quantification in different types of samples by a real-time PCR. Pol. J. Vet. Sci. $12,311-315$

McFarlane S, Aitken J, Sutherland JS, Nicholl MJ, Preston VG, Preston CM (2011): Early induction of autophagy in human fibroblasts after infection with human cytomegalovirus or herpes simplex virus 1. J. Virol. 85, 4212-4221. http://dx.doi.org/10.1128/JVI.02435-10

Nugent J, Birch-Machin I, Smith KC, Mumford JA, Swann Z, Newton JR, Bowden RJ, Allen GP, Davis-Poynter N (2006): Analysis of equid herpesvirus 1 strain variation reveals a point mutation of the DNA polymerase strongly associated with neuropathogenic versus nonneuropathogenic disease outbreaks. J. Virol. 80, 4047-4060. http:// dx.doi.org/10.1128/JVI.80.8.4047-4060.2006

Radtke K, English L, Rondeau C, Leib D, Lippé E, Desjardins M (2013): Inhibition of the host translation shutoff response by herpes simplex virus 1 triggers nuclearderived autophagy. J. Virol. 87, 3990-3997. http://dx.doi. org/10.1128/JVI.02974-12

Rasmussen SB, Horan KA, Holm C.K, Stranks AJ, Mettenleiter TC, Simon AC, Jensen SB, Rixon FJ, He B, Paludan SR: (2011): Activation of autophagy by alpha-herpesviruses is mediated by cytoplasmic viral DNA through a mechanism dependent on STING. J. Immunol. 187, 5268-5276. http://dx.doi.org/10.4049/jimmunol.1100949 